\section{The development of myasthe- nia gravis in a patient with facioscapulohumeral muscular dystrophy: case report and literature review}

Feryal Nauman, Muhammad Fawwad Ahmed Hussain, Ahmet Z. Burakgazi Neuroscience Section, Department of Medicine, Virginia Tech Carilion School of Medicine, Roanoke, VA, USA

\begin{abstract}
The co-existence of facioscapulohumeral muscle dystrophy (FSHD) and myasthenia gravis (MG) is very rare and few cases have been described in the literature. To increase the awareness of the health care providers, we present herein a rare case of MG in a patient with FSHD, discuss the diagnostic challenges, pre- and post-treatment findings and provide a literature review.
\end{abstract}

\section{Introduction}

Facioscapulohumeral muscular dystrophy (FSHD) is an autosomal dominant inherited form of muscular dystrophy with a reported prevalence ranging from 1 in 20,000 to 1 in $833.3 .1,2$ FSHD usually first presents with asymmetric and mild face muscle weakness that may go unrecognized at first. The most common initial clinical presentation is trouble with head and shoulder activities related to weakness of the neck and shoulder girdle muscles, later progressing to involve the truncal and lower extremity muscles. ${ }^{1}$ The bulbar and extraocular muscles are typically spared in FSHD. 3

Myasthenia gravis (MG) is a heterogeneous group of autoimmune diseases targeting the neuromuscular junction with the involvement of the extraocular and bulbar muscles. ${ }^{4}$ The existence of both FSHD and MG in same patient is very rare and few such cases have been reported in the literature. Their coexistence may be coincidental, but it has been postulated that it may be due to the production of acetylcholine receptor antibodies as a result of auto inflammation secondary to the muscle fiber degeneration of myopathies. 5 The coexistence of both pathologies provides diagnostic and management challenges for providers.

To increase the awareness of health care providers, we herein report on a rare case of MG in a patient with FSHD, discuss the diagnostic challenges, pre- and post-treatment findings and conduct a literature review.

\section{Case Report}

We present the case of a 77-year-old male who was previously diagnosed with FSHD at age 50 and who came to our clinic due to the worsening of new symptoms, including double vision, ptosis and swallowing issues, and the worsening of previous weaknesses. Along with FSHD, he was diagnosed with MG at age 74 .

When he was first diagnosed with FSHD, he presented with weakness of the facial muscles, proximal distal muscle weakness in the upper limbs and right $>$ left weakness in the lower limbs along with gait disturbance. The initial neurological examination in 2002 revealed weakness of his orbicularis oris muscles, neck flexion muscle (4/5), proximal muscle weakness $(4 / 5)$ including the deltoid and triceps muscles of the upper limbs, weakness of ankle dorsiflexion on the right (4-/5) and left (5-/5) and weakness of quadriceps (4/5) and hamstrings (5-/5) on both sides. He had a waddling gait at that time. Creatinine kinase and aldolase levels were elevated. The diagnosis of FSHD was confirmed with genetic and electrodiagnostic tests in 2002. The patient had progressive weakness and a decline in function over time. A follow up visit in 2015 at a local Muscular Dystrophy Association clinic revealed a remarkable decline in function. He had progressive weakness in lower $>$ upper limbs and facial muscle weakness. He had required the use of a wheelchair since 2013 and had greater difficulty in lifting, walking and performing fine motor activities. The follow up neuro exam in 2015 showed weakness of the deltoid $(3 / 5)$, triceps $(3 / 5)$, biceps (4-/5), pronator teres (4-/5), wrist flexion (4-/5), wrist extension (4-5), abductor pollicis brevis (4-/5), and interossei muscle (4-/5) on both sides and weakness of the right lower limb (1-2/5) and left lower limb, (4/5) including in the iliopsoas, adductor magnus, quadriceps, hamstrings, tibialis anterior and medial gastrocunemous muscles. The sensory examination was within normal limits. He was unable to walk due to worsening weakness and had required a wheelchair.

In 2016, he developed new-onset double vision, worsening facial and neck weakness and worsening weakness in swallowing in addition to generalized weakness. Since an involvement of extraocular muscles is rare in FSHD, an alternative diagno-
Correspondence: Ahmet Z. Burakgazi, Neuroscience Section, Department of Medicine, Virginia Tech Carilion School of Medicine, 3 Riverside Circle, Roanoke, VA 24016, USA

Tel.: +1.540-521-4592.

E-mail: drburakgazi@yahoo.com

Key words: facioscapulohumeral muscular dystrophy, myasthenia gravis.

Contributions: the authors contributed equally.

Conflict of interest: the authors declare no potential conflict of interest.

Funding: none.

Received for publication: 3 June 2019.

Accepted for publication: 10 June 2019.

This work is licensed under a Creative Commons Attribution NonCommercial 4.0 License (CC BY-NC 4.0).

(C) Copyright: the Author(s), 2019

Licensee PAGEPress, Italy

Neurology International 2019; 11:8191

doi:10.4081/ni.2019.8191

sis was investigated. A blood work up showed elevated acetylcholine receptor (AchR) antibody levels. Chest computed tomography was negative for thymus pathology. Due to uncontrolled diabetes, he was not a good candidate for steroids and started on pyridostigmine and mycophenolate mofetil. His MG related symptoms were resolved with this treatment. During the follow up course, he had a worsening of some symptoms, presenting with double vision, worsening of facial and neck weakness and difficulties in swallowing. He was treated with an intravenous immunoglobulin (IVIG) treatment $(1 \mathrm{gr} / \mathrm{kg} /$ day for 2 days). He showed a remarkable improvement in his symptoms with the IVIG treatments.

\section{Discussion}

FSHD is the third most common form of muscular dystrophy and has a predominantly autosomal dominant inheritance; however, sporadic cases (10-30\%) have also been seen. 6 The gene involved in FSHD is located on subtelomeric portion of chromosome 4 (4q35), which consists of a polymorphic repeat structure designated as D4Z4. The deletion of the D4Z4 repeat units leads to the development of FSHD and forms the basis for molecular diagnostic 
tests. Despite identifying the specific genetic defect, the pathophysiologic effects of this deletion are largely unknown. Mainly, it is the identification of the typical pattern of muscle involvement that makes a diagnosis for FSHD. FSHD is confirmed through a test of molecular DNA isolated from peripheral blood leucocytes with a specificity and sensitivity of $95 \%$. Due to the lack of a complete understanding of the pathophysiology of FSHD, treatment is mainly supportive therapy.7,8 The complexity of the genetics involved in this disorder has led to many studies and diagnostic tests that have revealed the co-existence of other myotonic dystrophies that warrant attention and further investigation.

There have been few previous reports of patients with a diagnosis of FSHD who were also found to have circulating antibodies against AchR. In 2002, McGonian et al. ${ }^{9}$ reported on a 56-year-old man who presented with ptosis that improved with edrophonium. MG was diagnosed based on large titers of AchR antibodies. In the same case, it was noticed that the patient also had a nearly 40-year history of foot drop and shoulder girdle weakness. Further electrodiagnostic and genetic tests revealed a diagnosis of FSHD.10 Similarly, Sansone et al. reported on a patient with known FSHD who presented with an acute onset of bulbar symptoms and ptosis. MG was diagnosed by testing positive for AchR antibodies. ${ }^{11}$ In 2012, Asadollahi et al. described a case of a 70 -year-old male with a 15-day history of difficulty chewing and dysarthria. MG was confirmed after the detection of AchR antibodies and nerve conduction studies. He also had a 50-year history of upper extremity weakness. A genetic analysis showed he had FSHD as well. ${ }^{9}$

As described above, our patient was diagnosed with FSHD about 25 years prior to the diagnosis with MG. He initially had a typical FSHD presentation and a progressive decline in his strength and function over time. When he developed an atypical presentation of FSHD, such as diplopia and extraocular muscle involvement, a significant worsening of neck muscle weakness and severe swallowing difficulties, alternative diagnoses were investigated that led to the discovery of the AchR antibodies. The patient was treated with an immunosuppressant and made a significant improvement in symptoms over time. He was treated with IVIG when he had MG exacerbation and made good improvement. It has been shown that patients with primary muscular disease rarely develop AchR antibodies that lead to the additional features of MG. The mechanism may work mainly through auto inflammation secondary to muscle degeneration that induces an innate immune response rather than an immune process in the thymus. 5

\section{Conclusions}

There have been few cases such as those mentioned above where FSHD and MG have existed concurrently. The purpose of highlighting them is to bring to the notice of physicians that if FSHD patients develop unusual symptoms, such as diplopia, extraocular muscle involvement and remarkable bulbar weakness, alternative diagnoses such as MG should be included in the differential diagnosis. Such symptoms should be sought carefully as early diagnosis can help in altering treatment and the plan of care.

\section{References}

1. Wang LH, Tawil R. Facioscapulohumeral Dystrophy. Curr Neurol Neurosci Rep 2016;16:66.

2. Deenen JC, Arnts H, van der Maarel $\mathrm{SM}$, et al. Population-based incidence and prevalence of facioscapulohumeral dystrophy. Neurology 2014:83:1056-9.

3. Tawil R, van der Maarel SM, Tapscott SJ. Facioscapulohumeral dystrophy: the path to consensus on pathophysiology. Skeletal Muscle 2014:10;4:12.

4. Sieb JP. Myasthenia gravis: an update for the clinician. Clin Exp Immunol 2014; 175:408-18.

5. Lane RJ, Roncarolia F, Charles P, et al. Acetylcholine receptor antibodies in patients with genetic myopathies: Clinical and biological significance. Neuromusc Disord 2012;22:122-8.

6. Zatz M1, Marie SK, Passos-Bueno MR, et al. High proportion of new mutations and possible anticipation in Brazilian facioscapulohumeral muscular dystrophy families. Am J Hum Genet 1995;56:99-105.

7. Pandya S, King WM, Tawil R. Facioscapulohumeral dystrophy. Phys Ther 2008;88:105-13.

8. Tawil R. Facioscapulohumeral muscular dystrophy. Neurotherapeutics 2008;5:601-6.

9. Asadollahi M, Rezaiyan B, Amjadi H. A rare case of facioscapulohumeral muscular dystrophy and myasthenia gravis. Iran J Neurol 2012;11:28-9.

10. McGonigal A, Thomas AM, Petty RK. Facioscapulohumeral muscular dystrophy and myasthenia gravis co-existing in the same patient: a case report. J Neurol 2002;249:219-20.

11. Sansone V, Saperstein DS, Barohn RJ, Meola G. Concurrence of facioscapulohumeral muscular dystrophy and myasthenia gravis. Muscle Nerve 2004;30:679-80.

12. Filippelli E, Barone S, Granata A, et al. A case of facioscapulohumeral muscular dystrophy and myasthenia gravis with positivity of anti-Ach receptor antibody: a fortuitous association? Neurol Sci 2019;40:195-7. 